\title{
Hair Follicle Infundibulum
}

National Cancer Institute

\section{Source}

National Cancer Institute. Hair Follicle Infundibulum. NCI Thesaurus. Code C32708.

The upper portion of a hair follicle extending from the sebaceous gland duct to the follicular orifice. 